\title{
Mixotrophic Growth of Astaxanthin-Rich Alga Haematococcus pluvialis using Refined Crude Glycerol as Carbon Substrate: Batch and Fed-Batch Cultivations
}

\author{
Pongsathorn DECHATIWONGSE ${ }^{1,2, *}$ and Wanna CHOORIT ${ }^{2,3}$ \\ ${ }^{1}$ Division of Chemical Engineering, School of Engineering and Technology, Walailak University, \\ Nakhon Si Thammarat 80160, Thailand \\ ${ }^{2}$ Biomass and Oil Palm Center of Excellence, Walailak University, \\ Nakhon Si Thammarat 80160, Thailand \\ ${ }^{3}$ Division of Biotechnology, School of Agricultural Technology, Walailak University, \\ Nakhon Si Thammarat 80160, Thailand
}

("Corresponding author's e-mail: pongsathorn.dechatiwongse@gmail.com)

Received: 21 August 2019, Revised: 8 November 2019, Accepted: 20 December 2019

\begin{abstract}
Due to a current overabundance of crude glycerol produced from the biodiesel industry, the compound has the potential to be used as an inexpensive carbon source for growing the green microalga Haematococcus pluvialis (H. pluvialis), the richest source of natural astaxanthin (ATX). In order to investigate the practical use of crude glycerol, microalgal cultures were grown mixotrophically and heterotrophically in BG11 medium with the supplementation of refined crude glycerol, a mixture of glycerol and ethanol, under 2, 5 and $7 \mathrm{~g} \mathrm{~L}^{-1}$, using photoautotrophic cultivation as a control. H. pluvialis green-stage growth and red-stage ATX accumulation were effectively facilitated by mixotrophic conditions, with the highest $\mu$ of $0.27 \pm 0.03$ day $^{-1}$ and the highest ATX content of $(3.5 \pm 0.4 \% \mathrm{wt})$ (both observed under $7 \mathrm{~g}$ glycerol $\mathrm{L}^{-1}$ ). In contrast, growth was completely inhibited under heterotrophic conditions. Under repeated fed-batch operation, the exponential growth phase, during green-stage mixotrophic cultivation, was significantly extended from 5 days (in batch) to 24 days, making biomass yield of $1.86 \pm 0.06 \mathrm{~g} \mathrm{DCW} \mathrm{L}^{-1}$ (around 2.6-fold higher). Monitoring of substrates (glycerol, ethanol and nitrate) in the broth was carried out and subsequently suggested that further optimization of media could be made.
\end{abstract}

Keywords: Astaxanthin, Fed-batch, Haematococcus pluvialis, Mixotrophic, Refined crude glycerol

$\begin{array}{ll}\text { Abbreviations } & \\ \text { ATX } & \text { Astaxanthin } \\ \mathrm{CaCl}_{2} \cdot 2 \mathrm{H}_{2} \mathrm{O} & \text { Calcium chloride dihydrate } \\ \mathrm{CO}_{2} & \text { Carbon dioxide } \\ \mathrm{CoCl}_{2} \cdot 6 \mathrm{H}_{2} \mathrm{O} & \text { Cobalt (II) chloride } \\ \mathrm{CuSO}_{4} \cdot 7 \mathrm{H}_{2} \mathrm{O} & \text { Copper sulfate heptahydrate } \\ \text { DCW } & \text { Dry cell weight } \\ \text { EDTA (disodium salt) } & \text { Ethylenediaminetetraacetic acid (disodium salt) } \\ \mathrm{H}_{3} \mathrm{BO}_{3} & \text { Boric acid } \\ \text { H. pluvialis } & \text { Haematococcus pluvialis } \\ \mathrm{H}_{2} \mathrm{SO}_{4} & \text { Sulfuric acid } \\ \mathrm{K}_{2} \mathrm{HPO}_{4} & \text { Potassium hydrogen phosphate }\end{array}$

Walailak J Sci \& Tech 2021; 18(2): 7354 


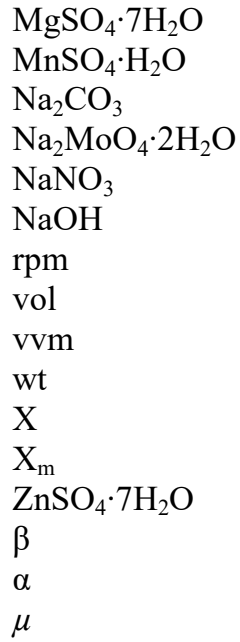

\author{
Magnesium sulfate heptahydrate \\ Manganese sulfate monohydrate \\ Sodium carbonate \\ Sodium molybdate dehydrate \\ Sodium nitrate \\ Sodium hydroxide \\ Revolution per minute \\ Volume \\ Volume of air per volume of medium per minute \\ Weight \\ Dry biomass concentration \\ Maximum biomass concentration \\ Zinc sulfate heptahydrate \\ Beta \\ Alpha \\ Specific growth rate
}

\section{Introduction}

Astaxanthin (ATX) is a renowned and superior antioxidant compound because it has more powerful antioxidant activity than that of other carotenoids, especially in quenching singlet oxygens and scavenging free radicals [1,2]. Because of its outstanding antioxidant nature, the potential of ATX in protecting against cancers [3-5], enhancing the human immune system [6,7], preventing the damaging effects of ultraviolet radiation [8-10], and treating a number of human diseases [11-13] have been reported. In addition to its health benefits, ATX also has a major application as a feed additive in aquaculture and the poultry industry $[14,15]$. In 2016, the global market value of ATX was reported to be 555.4 million US dollars and was forecasted to rise constantly until 2025 [16]. Currently, over $95 \%$ of ATX available on the market is chemically synthesized using petrochemicals due to cost-efficiency for mass production [17,18]. However, synthetic ATX has 20 times lower antioxidant capacity than its natural counterpart and, to date, has not been approved for human consumption [14,17,19]; consequently; recent interest in natural ATX has increased substantially. Natural ATX is commonly present as a redorange pigment in many aquatic animals, such as salmonids, shrimp, and crayfish. In fact, the compound is primarily biosynthesized by microalgae and phytoplankton, and consumed by fish, from where it is added to higher levels in the food chain [12].

Among natural sources of ATX, the freshwater, unicellular green microalga Haematococcus pluvialis (H. Pluvialis) is known to be the richest source of natural ATX (it can accumulate up to $5 \%$ of dry cell weight (DCW) [14,20]), and is currently grown on an industrial scale [21-23]. The cells are normally green, but under stress conditions, including intense light [24-26], high salinity [27], extreme $\mathrm{pH}[28,29]$, high temperature [30,31], and nutrient deprivation [32-34]), the green vegetative cells physically change into red cysts with a thick cell wall, indicating the formation of ATX. To date, various cultivation methods in photoautotrophic, heterotrophic, and mixotrophic growth conditions, using open raceway ponds or enclosed photobioreactors for culturing $H$. pluvialis, have been demonstrated [35-41]. Current commercial production of ATX by H. pluvialis employs a 2-stage process, in which vegetative green cells are primarily produced under optimal conditions in a nutrient-replete medium in enclosed photobioreactors (green-stage), before the culture is exposed to stress conditions (usually a combination of high irradiance and nitrogen deficiency) in open systems to induce ATX synthesis (red-stage) $[39,42,43]$.

Although mass cultivation of $H$. pluvialis for producing natural ATX has great potential and is an attractive business opportunity, microalgae-derived ATX only accounts for less than $1 \%$ of the commercialized quantity, due to the much lower price of synthetic ATX and several technological problems associated with the development of large-scale microalgae cultivation $[17,44,45]$. In order to 
make the product costs of ATX produced from H. pluvialis become more competitive with those of the synthetic alternative, it is very important to maximize vegetative cell densities of the alga during the green stage, as well as to maximize ATX production during the red stage [45]. At the same time, it is equally essential to minimize the costs associated with microalgal cultivation. For H.pluvialis cultivation, nutrients were estimated to be approximately $17 \%$ of the production costs [18]. Since carbon is one of essential macro-elements, accounting for a substantial part of microalgal biomass composition (46-53\% wt) [46], an approach worth investigating is the utilization of waste carbon in culturing H. pluvialis, which could help to decrease cultivation costs [45].

Glycerol $\left(\mathrm{C}_{3} \mathrm{H}_{8} \mathrm{O}_{3}\right)$ is a by-product of transesterification reaction in biodiesel production, with a stoichiometric share of $10 \% \mathrm{wt}[47,48]$. Biodiesel-derived glycerol is often referred to as crude glycerol, and is a mixture of glycerol, methanol, soap, salts, non-glycerol organic matter, and water [49]. Crude glycerol should be differentiated from technical-grade glycerol, which is a refined product with a high purity of $95 \%$ minimum [50], suitable for use in food, personal care, cosmetics, and pharmaceuticals $[51,52]$. The dramatic expansion of the global biodiesel industry has created an overabundance of glycerol that has caused a more than 10-fold decrease in crude glycerol prices in recent years [53]. The purification of crude glycerol into the technical grade is an expensive process; hence, almost half of the total crude glycerol generated (around 2 billion pounds each year) is subjected to landfill disposal [53,54], which has generated serious threats to the environment As a result, numerous studies have been made on value-added utilization of crude glycerol from biodiesel production, through both biological and chemical conversions $[47,49,53,55,56]$. The use of crude glycerol for culturing various strains of microalgae for the purpose of enhancing biomass and bioproduct production has been well-demonstrated [57-61]. For $H$. pluvialis, although the microalga has been previously reported to grow using a number of organic carbon substrates [38,62-70], there have only been a few studies on microalgal cultivation using glycerol [68,71]. Therefore, current limited knowledge of the use of biodiesel-derived glycerol for cultivating the green alga $H$. pluvialis has led to the conduction of this study, which aims to: 1 ) investigate the possibility of using biodiesel-derived glycerol as an organic carbon source, facilitating the growth of $H$. pluvialis batch culture under heterotrophic and mixotrophic conditions (the autotrophic growth condition was used as the control condition); 2) examine ATX accumulation in H. pluvialis cells, produced from different greenstage growth conditions, and 3) to investigate the use of repeated fed-batch cultivation as a tool to enhance the growth and ATX production of $H$. pluvialis. For the last objective, fed-batch cultivation was investigated as it was known for its superior ability in enhancing $H$. pluvialis growth (compared with batch cultivation), with an ease of implementation and operation ability $[35,72,73]$. The outcome of this study could be used for the further design of large-scale cultivation methods for biomass and ATX production from $H$. pluvialis. isposal of crude glycerol tainted with salt, methanol, free fatty acids, etc., causes severe economic and environmental challenge, disposal of crude glycerol tainted with salt, methanol, free fatty acids, etc., causes severe economic and environmental challeng.

\section{Materials and methods}

Refined crude glycerol preparation

Crude glycerol used in this study was kindly provided by Assoc. Prof. Dr. Chakrit Tongurai, Department of Chemical Engineering, Prince of Songkla University, Thailand. However, the crude glycerol could not be used directly for culturing the microalga $H$. pluvialis (detailed reasons given in Results and Discussions), due to the sample's toxic impurities. As a result, purification of crude glycerol to produce a more refined glycerol with higher purity was performed by adapting the method of Manosak et al. [74], as illustrated in Figure 1. Briefly, crude glycerol was acidified by the addition of $98 \%$ sulfuric acid to the desired $\mathrm{pH}(2-3)$, and then left for $12 \mathrm{~h}$ until the solution was separated into 2 distinct layers, where the upper layer was a free fatty acid and the bottom layer was glycerol-rich and aqueous. Subsequently, the glycerol-rich layer was collected and neutralized by the addition of $5 \mathrm{M} \mathrm{NaOH}$ to $\mathrm{pH}$ 7.0 , left for $30 \mathrm{~min}$, and then filtered to remove the precipitated salt. The glycerol-rich layer was mixed with ethanol at a solvent: Glycerol ratio of 1:1 ( $\mathrm{vol} / \mathrm{vol}$ ), shaken for $30 \mathrm{~min}$, and then left for $1 \mathrm{~h}$ to precipitate the impurities as salt. Two layers formed, with the glycerol-alcohol phase on the top and the 
impurity salts on the bottom. The upper layer was separated by slow decantation to eliminate the salts. Refined crude glycerol was then obtained after autoclaving at $121{ }^{\circ} \mathrm{C}$ for $15 \mathrm{~min}$. By using high performance liquid chromatography (HPLC), the organic carbon components within the crude and refined crude glycerol samples were determined and are tabulated in Table 1. It can be seen from the table that our refined crude glycerol was a mixture of glycerol and ethanol.

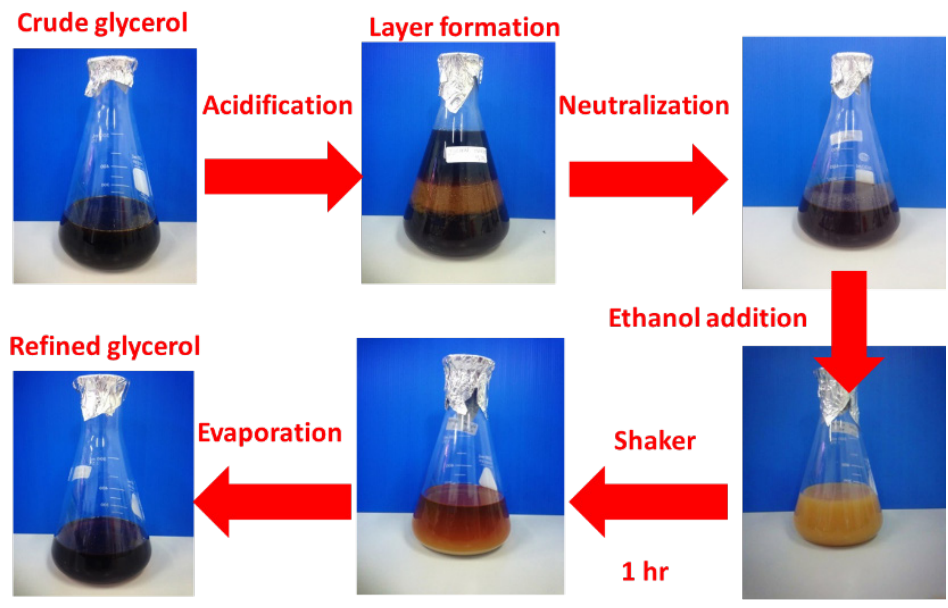

Figure 1 Schematic presentation of crude glycerol purification method, adapted from Manosak et al. [74].

Table 1 Organic carbon components of crude and refined crude glycerol samples.

\begin{tabular}{ccc}
\hline Compound & Crude glycerol $\left(\mathbf{g ~ L}^{\mathbf{- 1}}\right)$ & Refined crude glycerol $\mathbf{( \mathbf { g ~ L } ^ { \mathbf { - 1 } } )}$ \\
\hline Glycerol & $257.3 \pm 3.3$ & $497.3 \pm 7.7$ \\
Methanol & $74.2 \pm 2.5$ & None \\
Ethanol & None & $110.5 \pm 4.2$ \\
\hline
\end{tabular}

\section{Microalgal strain}

In this study, H. pluvialis K-0084 (onwards referred to as $H$. pluvialis) was selected as the model microorganism, as it is a widely-studied strain. The microalgal culture was purchased from the Scandinavian Culture Collection for Algae \& Protozoa, University of Copenhagen, Denmark. Pre-culture was grown in a $250 \mathrm{~mL}$ Erlenmeyer flask containing $100 \mathrm{~mL}$ of BG-11 medium [75] under continuous illumination provided by a fluorescent cool white lamp at a light intensity of 4,000 lux [76]. For the preparation of the inoculum, $100 \mathrm{~mL}$ of pre-culture was transferred and incubated autotrophically in a $1 \mathrm{~L}$ Duran bottle containing $700 \mathrm{~mL}$ of fresh BG-11 medium under continuous illumination at $25^{\circ} \mathrm{C}$ for 14 days [76]. A flow rate of $0.5 \mathrm{vvm}$ of $1 \% \mathrm{CO}_{2}$ in air was supplied continuously to the culture in order to provide an inorganic carbon source. A 14 day old culture was collected by centrifugation at 3,500 rpm for $15 \mathrm{~min}$ and the supernatant was removed. The cell pellet was washed twice with $0.85 \% \mathrm{wt} \mathrm{vol}^{-1} \mathrm{NaCl}$ and then resuspended in the BG-11 medium and used as inoculum in the experiment.

\section{Medium composition}

The BG-11 medium [75] was composed of the following components (per L of distilled water): 1.5 $\mathrm{g} \mathrm{NaNO}_{3}, 0.04 \mathrm{~g} \mathrm{~K}_{2} \mathrm{HPO}_{4}, 0.02 \mathrm{~g} \mathrm{Na}_{2} \mathrm{CO}_{3}, 0.003 \mathrm{~g}$ ferric ammonium citrate, $0.003 \mathrm{~g}$ citric acid, $0.001 \mathrm{~g}$ EDTA (disodium salt), $0.075 \mathrm{~g} \mathrm{MgSO} \cdot 7 \mathrm{H}_{2} \mathrm{O}, 0.036 \mathrm{~g} \mathrm{CaCl}_{2} \cdot 2 \mathrm{H}_{2} \mathrm{O}$, and $1 \mathrm{~mL}$ trace metal mix A5. Trace metal mix A5 consisted of $\mathrm{H}_{3} \mathrm{BO}_{3} 2.86 \mathrm{~g} \mathrm{~L}^{-1}, \mathrm{MnSO}_{4} \cdot \mathrm{H}_{2} \mathrm{O} 1.48 \mathrm{~g} \mathrm{~L}^{-1}, \mathrm{ZnSO}_{4} \cdot 7 \mathrm{H}_{2} \mathrm{O} 0.222 \mathrm{~g} \mathrm{~L}^{-1}$, 
$\mathrm{Na}_{2} \mathrm{MoO}_{4} \cdot 2 \mathrm{H}_{2} \mathrm{O} 0.39 \mathrm{~g} \mathrm{~L}^{-1}, \mathrm{CuSO}_{4} \cdot 7 \mathrm{H}_{2} \mathrm{O} 0.079 \mathrm{~g} \mathrm{~L}^{-1}$, and $\mathrm{CoCl}_{2} \cdot 6 \mathrm{H}_{2} \mathrm{O} 40.4 \mathrm{mg} \mathrm{L}^{-1}$. For both mixotrophic and heterotrophic cultivation, refined crude glycerol was used as an organic carbon source. The concentration of refined crude glycerol was varied depending on the experiment by adding the refined crude glycerol to the BG-11 medium to achieve the desired glycerol concentration $\left(2,5\right.$ and $\left.7 \mathrm{~g} \mathrm{~L}^{-1}\right)$. The medium was then adjusted to $\mathrm{pH} 7.2$ and autoclaved at $121{ }^{\circ} \mathrm{C}$ for $15 \mathrm{~min}$ before use.

\section{Batch cultivation}

Both photoautotrophic and mixotrophic growths of $H$. pluvialis cultures were carried out in a $1 \mathrm{~L}$ Duran bottle containing $700 \mathrm{~mL}$ of liquid culture. The culture was maintained at a temperature of $25^{\circ} \mathrm{C}$. Continuous illumination was provided by a cool white fluorescent lamp $(18 \mathrm{~W})$ at a light intensity of 54 $\mu \mathrm{mol} \mathrm{m} \mathrm{m}^{-1}$ [76]. The gas flow of $1 \% \mathrm{CO}_{2}$ in air was continuously supplied to the culture at a flow rate of $0.5 \mathrm{vvm}$. Agitation provided by a magnetic stirrer was made in order to ensure a well-mixed condition within the liquid culture. For heterotrohic growth, cultivation was also conducted in a 1 L Duran bottle, which was then covered with an aluminum foil to provide dark conditions, and only air was supplied to the culture at the same flowrate. The total experimental period for all batch cultivations was set to be 6 days. To minimize the risk of contamination in mixotrophic and heterotrophic cultivations, griseofulvin at a concentration of $0.1 \mathrm{~g} \mathrm{~L}^{-1}$ was added into the cultures. DCW and remaining substrates were determined daily. Three replications were performed for each experiment.

\section{Repeated fed-batch cultivation}

Repeated fed-batch cultivation was carried out in a $1 \mathrm{~L}$ Duran bottle containing $800 \mathrm{~mL}$ of liquid culture. The culture was grown in $2 \mathrm{~g} \mathrm{~L}^{-1}$ refined crude glycerol-supplemented BG11 medium with an initial biomass concentration of $0.2 \mathrm{~g} \mathrm{~L}^{-1}$, under light conditions provided by a fluorescent lamp at an intensity of 4,000 lux and a temperature of $25^{\circ} \mathrm{C}$. The gas flow of $1 \% \mathrm{CO}_{2}$ in air was supplied at a flow rate of $0.5 \mathrm{vvm}$. Agitation provided by a magnetic stirrer was made in order to ensure a well-mixed condition within the liquid culture. To initiate fed-batch operation, $200 \mathrm{~mL}$ of liquid culture was replaced by prepared feeding medium, containing refined crude glycerol at an approximate concentration of 15.3 $\mathrm{mL} \mathrm{L}{ }^{-1}$. The replacement was repeated every 6 days. It should be mentioned that, in this study, the term "cycle" was used to describe a period of fed-batch cultivation, with each period lasting for 6 days. Specifically, cycle 1 referred to the period starting from day 0 to day 6 (before the replacement was made), and then cycle 2 was for the subsequent period, starting from day 6 (after the replacement was made) to day 12, and so on. The cultivation was operated for 24 days. To minimize the risk of contamination, griseofulvin was also added into the culture at a concentration of $0.1 \mathrm{~g} / \mathrm{L}$. DCW and remaining substrates were determined daily. Three replications were performed for each experiment.

\section{ATX accumulation}

The vegetative cell culture of $50 \mathrm{~mL}$, produced from growth conditions investigated in this study, was collected, centrifuged, and washed twice with $0.89 \%$ sodium chloride. Then, cell pellets were resuspended in $800 \mathrm{~mL}$ of distilled water, in which nutrient-depleted conditions were provided for inducing ATX accumulation. Initial biomass concentrations of all conditions were adjusted to be $0.5 \mathrm{~g} \mathrm{~L}^{-1}$. The cultures were maintained under constant temperatures of $25^{\circ} \mathrm{C}$ and continuous illumination, provided by a cool white fluorescent lamp $(18 \mathrm{~W})$ at a light intensity of 10,000 lux [24-26]. The gas flow of $1.5 \%$ $\mathrm{CO}_{2}$ in air was continuously supplied to the culture at a flow rate of $0.5 \mathrm{vvm}$. For vegetative cells taken from batch cultures, the cells were collected on the last day of the growth experiment, while the ATX production experiment was allowed for 8 days. DCW was determined every 2 days, while ATX quantification was made on days $2,4,6$, and 8 . In the cases of fed-batch cultures, the cells were collected on the last day of each cycle during the growth experiment, while the ATX production was allowed for 7 days. DCW was determined on days 0, 2, 4, 6 and 7, while ATX quantification was only made on day 7. Three replications were performed for each experiment.

\section{Analytical methods}

DCW was determined by gravimetric analysis. A $10 \mathrm{~mL}$ sample of culture was collected, centrifuged at 5,000 rpm for $10 \mathrm{~min}$, washed twice with distilled water, and then dried at $105{ }^{\circ} \mathrm{C}$ until 
constant weight was obtained. Besides the biomass measurement, cell morphology during cultivation was monitored using a camera system (Canon EOS7000, Japan) mounted on a microscope (Nikon model ECLIPSE Ci-S, China). Medium $\mathrm{pH}$ was measured by a $\mathrm{pH}$ meter. The residual glycerol was determined using an HPLC system (model 2690, Waters, MA, USA) with a refractive index detector (model 2414, Waters, MA, USA) and a Metacarb H Plus $300 \times 8.0 \mathrm{~mm}^{2}$ column (Varian, CA, USA). The run conditions were as follows: a column temperature of $50^{\circ} \mathrm{C}$, a mobile phase of $0.01 \mathrm{M} \mathrm{H}_{2} \mathrm{SO}_{4}$, a flow rate of $0.3 \mathrm{~mL}$ $\mathrm{min}^{-1}$, and an injection volume of $20 \mu \mathrm{L}$. The amount of nitrate was measured by flow injection analysis (FIA) using FIAstarTM 5000 version 1.3. The pigments were extracted using a modified method of Sarada et al. [77]. In brief, $10 \mathrm{mg}$ of a sample cell was treated with $1 \mathrm{~mL}$ of $2 \mathrm{~N}$ hydrochloric acid at $70{ }^{\circ} \mathrm{C}$ for $10 \mathrm{~min}$. The sample was then cooled, centrifuged at 5,000 rpm for $10 \mathrm{~min}$, and washed twice with distilled water. Then, the sample was treated with acetone for $1 \mathrm{~h}$, and the extraction procedure was repeated until the pellet became white. The extract was evaporated under nitrogen gas to eliminate acetone. The dried pellet was mixed with $2 \mathrm{~mL}$ of $20 \mathrm{mM} \mathrm{NaOH}$ in methanol and kept overnight under nitrogen in darkness at $4{ }^{\circ} \mathrm{C}$ for the saponification of ATX esters. After saponification, the ATX concentration within the mixture was further analyzed using the HPLC system equipped with a reverse phase column (Zorbax-300SB, $4.6 \times 250 \mathrm{~mm}^{2}$, Agilent Technologies, USA). The mobile phase was $90 \%$ acetone with a flow rate of $0.8 \mathrm{~mL} \mathrm{~min}^{-1}$ at $40{ }^{\circ} \mathrm{C}$. The absorbance of the sample was measured at $470 \mathrm{~nm}$ with a photodiode array detector. Authentic free ATX, purchased from Sigma-Aldrich, was used as a standard.

\section{Results and discussion}

Effects of growth conditions and refined crude glycerol concentrations on $H$. pluvialis biomass production in batch cultivation

Although mixotrophic cultivation of some microalgae using crude glycerol as organic carbon source were previously demonstrated $[58,59,68,78,79]$, in this study, when BG11 medium supplemented with crude glycerol concentration of $2 \mathrm{~g} \mathrm{~L}^{-1}$ was used for culturing $H$. pluvialis, there was no apparent growth of the culture. This observation differed from the report by Andruleviciute et al. [68], which showed an enhancement in the growth of $H$. pluvialis culture by the supplement of technical glycerol at 2,5 and $10 \mathrm{~g}$ $\mathrm{L}^{-1}$. The possible reason for explaining this discrepancy was due to the variation in the composition of crude glycerol. Andruleviciute et al. [68] stated that their sample of crude glycerol was composed of 75 $80 \% \mathrm{wt}$ glycerol (with no statement given on the quantity of other impurities present), whereas in our sample, the sample contained only $\sim 24 \%$ wt glycerol (the other impurities being $\sim 7 \%$ wt methanol, $\sim 50$ $\%$ wt MONG (matter organic non-glycerol), $\sim 15 \%$ wt water, and $\sim 4 \%$ wt ash). Therefore, the growth cessation observed in our study most likely resulted from the higher level of impurities [47,80]. As a result, the treatment of crude glycerol was made in order to produce a more purified glycerol, which was termed as "refined crude glycerol". The sample was found to be a mixture of glycerol and ethanol, while methanol formerly present was completely removed (Table 1).

To investigate the influence of refined crude glycerol on $H$. pluvialis biomass production, microalgal cultures were mixotrophically and heterotrophically grown in BG11 medium and supplemented with refined crude glycerol at final concentrations of 2,5 and $7 \mathrm{~g} \mathrm{~L}^{-1}$, while photoautotrophically-grown culture was used as a control. The growth comparison is illustrated in Figure $\mathbf{2 A}$, which shows that, under our experimental specifications, the green alga $H$. pluvialis could grow effectively under photoautotrophic and mixotrophic conditions, but there was virtually no growth under all heterotrophic conditions. Our results were similar to previous studies [68,71], which reported enhanced mixotrophic growth in the presence of glycerol. For heterotrophic conditions, the apparent growth of microalga was observed when cultures were supplemented with acetate; however, there has been no previous investigation on the use of glycerol. Consequently, this study reveals the impractical use of glycerol for heterotrophically growing $H$. pluvialis. It can be also seen from Figure $2 \mathbf{A}$ that, for mixotrophic conditions, biomass concentrations increased from initial values of $0.13 \pm 0.00-0.21 \pm 0.04$ g DCW L ${ }^{-1}$ and reached maximum values on day 5, which was regarded as the beginning of the cell stationary phase. The maximum concentration increased with increasing concentrations of glycerol. The 
highest value of maximum biomass concentration $\left(\mathrm{X}_{\mathrm{m}}\right)$ of $0.71 \pm 0.03 \mathrm{~g} \mathrm{DCW} \mathrm{L}{ }^{-1}$, as well as a biomass increment $(\triangle \mathrm{X})$ of $0.56 \pm 0.03 \mathrm{~g} \mathrm{DCW} \mathrm{L}^{-1}$, were observed from the $H$. pluvialis culture, supplemented with $7 \mathrm{~g}$ glycerol $\mathrm{L}^{-1}$, while the values observed from both 2 and $5 \mathrm{~g}$ glycerol $\mathrm{L}^{-1}$ supplemented microalgal cultures were relatively similar and around 1.2 times lower. For photoautotrophically-grown culture, the maximum biomass concentration, also observed on day 5 , was $0.50 \pm 0.01 \mathrm{~g} \mathrm{DCW} \mathrm{L}^{-1}$, which is approximately 1.2 - 1.4 times lower than that of mixotrophically-grown cultures. A similar value of factor was reported by Azizi et al. [71]. These observations were commonly expected, since mixotrophicallygrown cultures could utilize refined crude glycerol that was present, in addition to $\mathrm{CO}_{2}$, in promoting biomass accumulation. In contrast with biomass yield, values of specific growth rate $(\mu)$ obtained under photoautotrophic and mixotrophic conditions were similar and in the range of $0.21 \pm 0.05-0.27 \pm 0.03$ day $^{-1}$. Our obtained rates are in the same order as those observed from mixotrophic cultivations using technical-grade glycerol with a purity of $75-80 \%$ [68], implying the practical utilization of refined crude glycerol produced in our laboratory. Nevertheless, these rates are around $50 \%$ less than the rate reported [71] when chemical-grade glycerol was used.

Regarding the medium $\mathrm{pH}$, Figure 2B shows similar trends of the $\mathrm{pH}$ profiles for photoautotrophically and mixotrophically grown cultures, regardless of glycerol concentration. Specifically, $\mathrm{pH}$ experienced a rapid and significant drop from initial values of $7.43 \pm 0.01$ to $8.04 \pm 0.04$ to the lowest value of $6.50 \pm 0.04$ on day 1 , then constantly rose to reach the final $\mathrm{pH}$ value of 7 on day 4 , and eventually remained constant until the end of the experiments. In contrast, for heterotrophic growth conditions, the initial drop was slight, and $\mathrm{pH}$ values remained almost constant until day 6 . As both photoautotrophic and mixotrophic conditions had the employment of $\mathrm{CO}_{2}$ in common, their larger initial $\mathrm{pH}$ drops could be caused by the acidic nature of dissolved $\mathrm{CO}_{2}$. During the first $24 \mathrm{~h}$, cells were still in an adaptation period, and would not fully carry out photosynthesis. The subsequent rise in $\mathrm{pH}$ value after day 1 then implied active photosynthesis and concurrent $\mathrm{CO}_{2}$ fixation [29].

In terms of substrates, substantial decrease in glycerol, ethanol, and nitrate concentrations were

clearly observed from mixotrophically-grown cultures (Figures $\mathbf{3 A}$ and 4), whereas, for heterotrophically-grown cultures, concentrations appeared to be relatively constant (Figures 3B and Figure 4). The latter observation could simply be the consequence of microalgal growth cessation, which led to virtually no consumption of nutrients in the broth. Although total ethanol consumption was found to be similar for all mixotrophic experiments $(\sim 60-64 \%)$, there was a great variation in the total glycerol consumption (Table 2). Especially, the highest value of glycerol consumption $(\sim 72 \%)$ was observed from $2 \mathrm{~g}$ glycerol L $\mathrm{L}^{-1}$ supplemented culture, and was approximately 2.4- and 5.5-fold higher those that of 5 and $7 \mathrm{~g}$ glycerol $\mathrm{L}^{-1}$ cultivations, respectively. Interestingly, our results showed the "diauxic growth behavior" of $H$. pluvialis to simultaneously utilize 2 organic carbon sources, which were ethanol and glycerol, for biomass production [81,82]. For nitrate consumption, it can be seen from Table 2 that only about $11-20 \%$ of the total supply was consumed by microalgal cultures, implying its initial quantity was also excessive. As a result, further significant reduction in both carbon and nitrogen substrates could be made in order to lower the cost of chemicals used in medium preparation. 

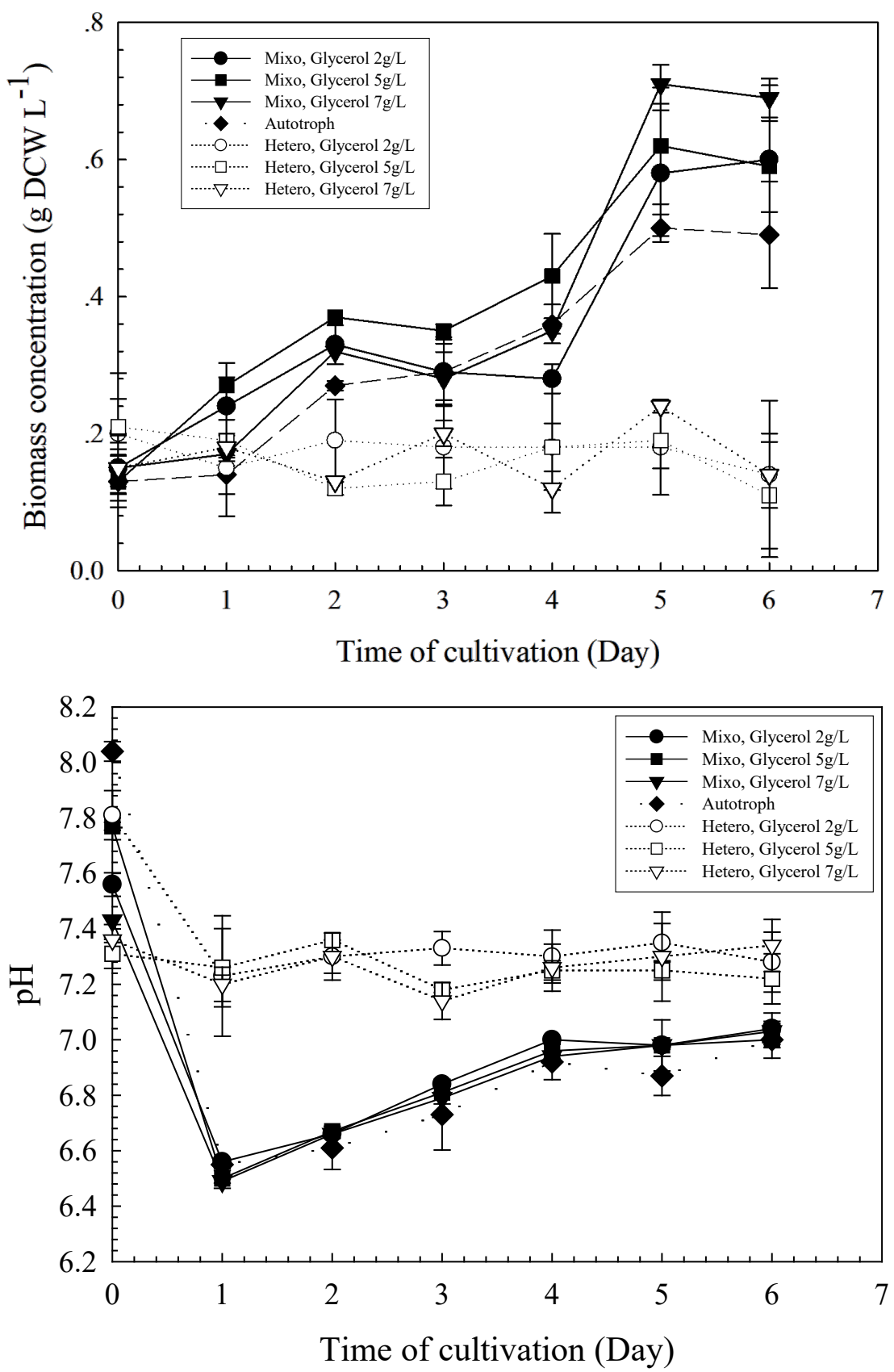

Figure 2 Time profiles of green-stage biomass concentration (A) and $\mathrm{pH}(\mathbf{B})$ of H. pluvialis cultures grown mixotrophically, photoautotrophically, and heterotrophically under refined crude glycerol concentrations of 2, 5 and $7 \mathrm{~g} \mathrm{~L}^{-1}$. 

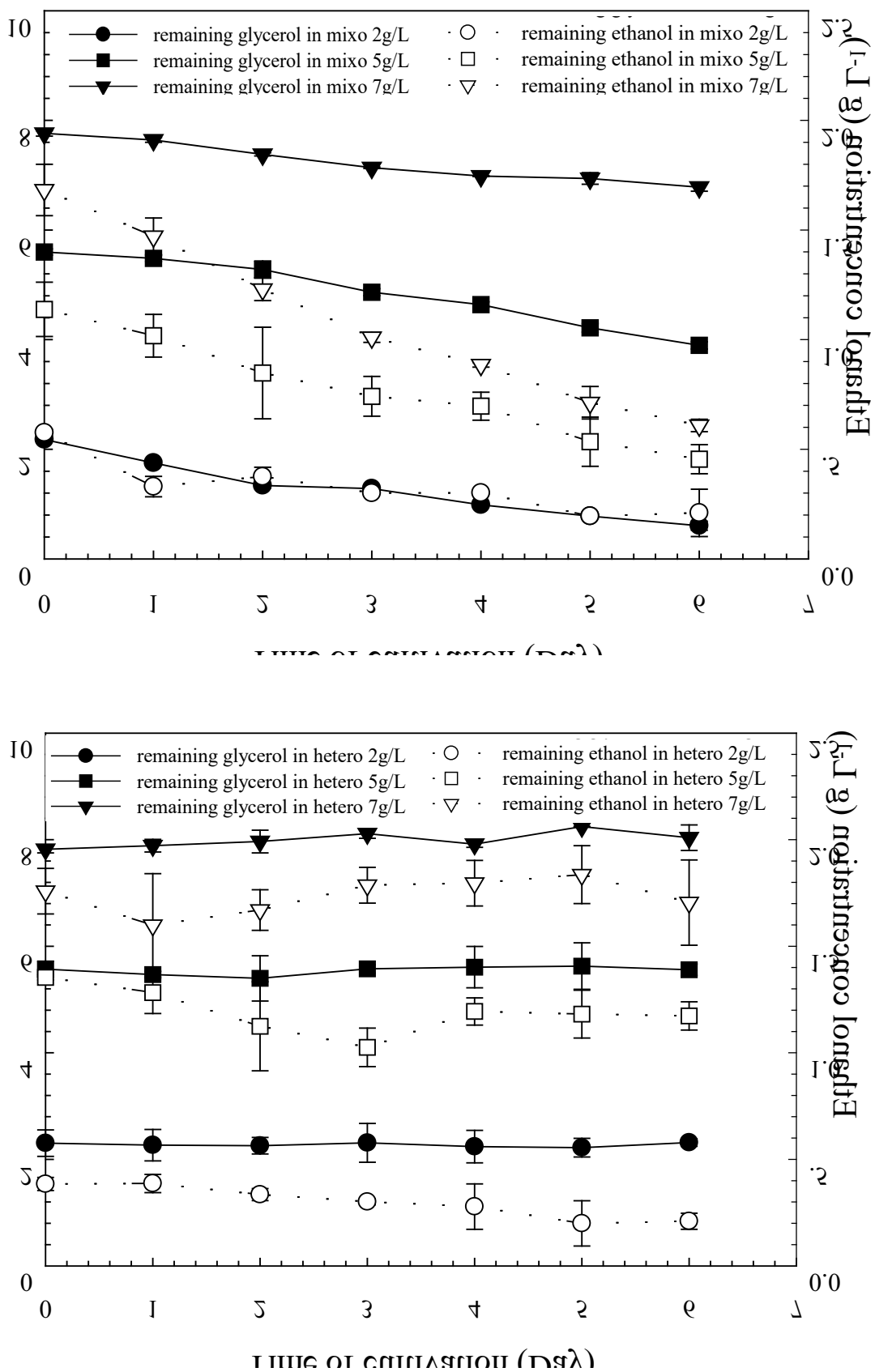

Figure 3 Time profiles of remaining glycerol and ethanol, during green-stage, in mixotrophic (A) and heterotrophic (B) cultivations of $H$. pluvialis under refined crude glycerol concentrations of 2,5 and $7 \mathrm{~g} \mathrm{~L}^{-1}$. 


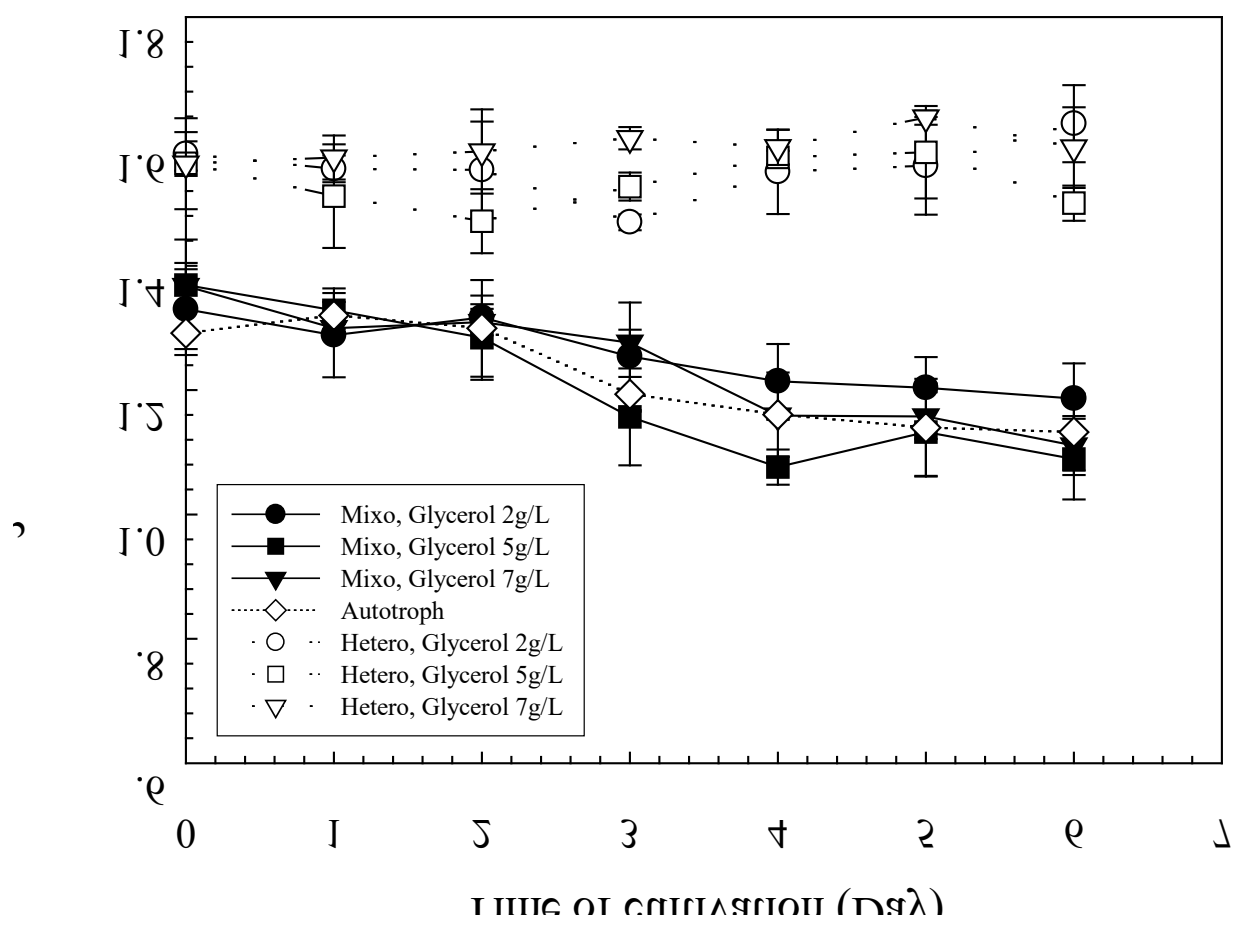

Figure 4 Time profile of remaining nitrate, during green-stage, in mixotrophic, photoautotrophic, and heterotrophic cultivations of $H$. pluvialis under refined crude glycerol concentrations of 2,5 and $7 \mathrm{~g} \mathrm{~L}^{-1}$.

Table 2 Effect of initial refined crude glycerol concentration on biomass and ATX production and substrate consumption of $H$. pluvialis batch cultures.

\begin{tabular}{|c|c|c|c|c|}
\hline \multirow{2}{*}{ Parameters } & \multirow{2}{*}{ Photoautotrophic } & \multicolumn{3}{|c|}{ Mixotrophic } \\
\hline & & 2 g glycerol $\mathrm{L}^{-1}$ & 5 g glycerol $\mathrm{L}^{-1}$ & $7{\text { g glycerol } \mathrm{L}^{-1}}^{-1}$ \\
\hline \multicolumn{5}{|c|}{ Green-stage } \\
\hline $\mathrm{X}_{\mathrm{m}}\left(\mathrm{g} \mathrm{DCW} \mathrm{L}^{-1}\right)$ & $0.50 \pm 0.01$ & $0.60 \pm 0.05$ & $0.62 \pm 0.05$ & $0.71 \pm 0.03$ \\
\hline$\Delta \mathrm{X}\left(\mathrm{g} \mathrm{DCW} \mathrm{L}^{-1}\right)$ & $0.37 \pm 0.01$ & $0.47 \pm 0.05$ & $0.48 \pm 0.05$ & $0.56 \pm 0.03$ \\
\hline$\mu\left(\right.$ day $\left.^{-1}\right)$ & $0.24 \pm 0.01$ & $0.21 \pm 0.05$ & $0.23 \pm 0.05$ & $0.27 \pm 0.03$ \\
\hline$\% \mathrm{NaNO}_{3}$ consumption & $12.3 \pm 1.6$ & $10.8 \pm 0.5$ & $20.4 \pm 1.2$ & $18.8 \pm 2.5$ \\
\hline$\%$ Glycerol consumption & - & $72.2 \pm 0.7$ & $30.4 \pm 7.2$ & $12.7 \pm 4.9$ \\
\hline$\%$ Ethanol consumption & - & $63.5 \pm 19.6$ & $60.0 \pm 1.6$ & $63.8 \pm 0.9$ \\
\hline \multicolumn{5}{|c|}{ Red-stage } \\
\hline $\mathrm{X}_{\mathrm{m}}\left(\mathrm{g} \mathrm{DCW} \mathrm{L}^{-1}\right)$ & $0.29 \pm 0.03$ & $0.56 \pm 0.02$ & $0.75 \pm 0.01$ & $0.56 \pm 0.00$ \\
\hline$\Delta \mathrm{X}\left(\mathrm{g} \mathrm{DCW} \mathrm{L}^{-1}\right)$ & $0.15 \pm 0.03$ & $0.32 \pm 0.00$ & $0.57 \pm 0.04$ & $0.45 \pm 0.04$ \\
\hline ATX content $(\% \mathrm{wt})$ & $2.7 \pm 0.5$ & $2.0 \pm 0.6$ & $1.9 \pm 0.1$ & $3.5 \pm 0.4$ \\
\hline
\end{tabular}

Data shown are the mean $\pm \mathrm{SD}$ of 3 independent measurements. 
Effects of growth conditions and refined crude glycerol concentrations on $H$. pluvialis ATX accumulation in batch cultivation

Although an enhancement in ATX accumulation by supplementation of organic carbon sources has been previously demonstrated, most studies were limited to the use of acetate $[65,69-71,83]$. Therefore, we investigated biomass and ATX production during red-stage of $H$. pluvlias by exposing vegetative cells collected from different green-stage growth conditions to intense irradiance and nutrient-depleted conditions (Figure 5). Evidently, the highest value of final biomass concentration of $0.75 \pm 0.01 \mathrm{~g}$ DCW $\mathrm{L}^{-1}$ was observed on day 6 from vegetative cells mixotrophically cultivated in the presence of $5 \mathrm{~g} \mathrm{~L}^{-1}$ refined crude glycerol (Figure 5A). This obtained value was around 1.3- and 2.6-fold higher than those obtained from the rest of mixotrophic and photoautotrophic conditions, respectively (Table 2). Nevertheless, the highest value of ATX content $(3.5 \pm 0.4 \% \mathrm{wt})$ was achieved with culture formerly cultivated using $7 \mathrm{~g} \mathrm{~L}^{-1}$ of refined crude glycerol. This obtained value was very similar to a previous study [71] which reported glycerol as the most promising substrate among 14 carbon sources in promoting $H$. pluvialis ATX production, and in the same order as many mixotrophic cultivations $[45,69,72,84,85]$. In addition, it can also be seen from the Table 2 that, only in presence of $7 \mathrm{~g} \mathrm{~L}^{-1}$ refined crude glycerol, $H$. pluvialis displayed an upward trend in ATX production until the end of the experiment (Figure 5B). In the other conditions, ATX level reached its peak on day 6 before it significantly dropped to the minimum on day 8. Our results also showed that, under photoautotrophic conditions, microalgal culture was able to accumulate subsequent ATX $(2.7 \pm 0.5 \% \mathrm{wt})$ of around 1.35 - 1.42-old higher than those of mixotrophic cultivations $\left(2.0 \pm 0.5\right.$ and $1.9 \pm 0.1 \%$ wt for 2 and $5 \mathrm{~g} \mathrm{~L}^{-1}$ refined crude glycerol, respectively). The latter observation could reasonably be explained by the photoautotrophic cultures producing much lower biomass concentrations, thereby having higher light penetration throughout, and thus higher light exposure of the cells [24-26]. On the other hand, the highest level of ATX under $7 \mathrm{~g} \mathrm{~L}^{-1}$ mixotrophically grown culture was most likely attributed by the highest quantities of glycerol and ethanol present in supplied refined crude glycerol. The former was previously reported to greatly enhance the accumulation of lipids within $H$. pluvialis cells [68], which is necessary for ATX production $[45,86]$, while the positive effect of the latter upon ATX accumulation was also suggested [87].

\section{Repeated fed-batch mixotrophic cultivation for $H$. pluvialis biomass and ATX production}

Fed-batch mixotrophic cultivations of $H$. pluvialis using acetate as organic carbon was previously demonstrated and found to effectively facilitate microalgal growth and ATX production [72,73]. As glycerol was shown to be superior to acetate in promoting microalgal biomass and ATX production (this study and [68,71]), a combination of glycerol and fed-batch operation for growing microalga was worth investigating. Since our results (Figure 3) indicated considerable amounts of remaining organic carbon substrates, when mixotrophically-grown cultures were supplemented with 5 and $7 \mathrm{~g}$ glycerol $\mathrm{L}^{-1}$, consequently, $2 \mathrm{~g}$ glycerol $\mathrm{L}^{-1}$ was selected as the initial concentration in the fed-batch culture, as well as the condition for the feeding. This justification was made in order to reduce the quantity of leftover substrates and subsequently prevent their accumulation, which may have had an inhibitory effect on microalgal growth [68]. 

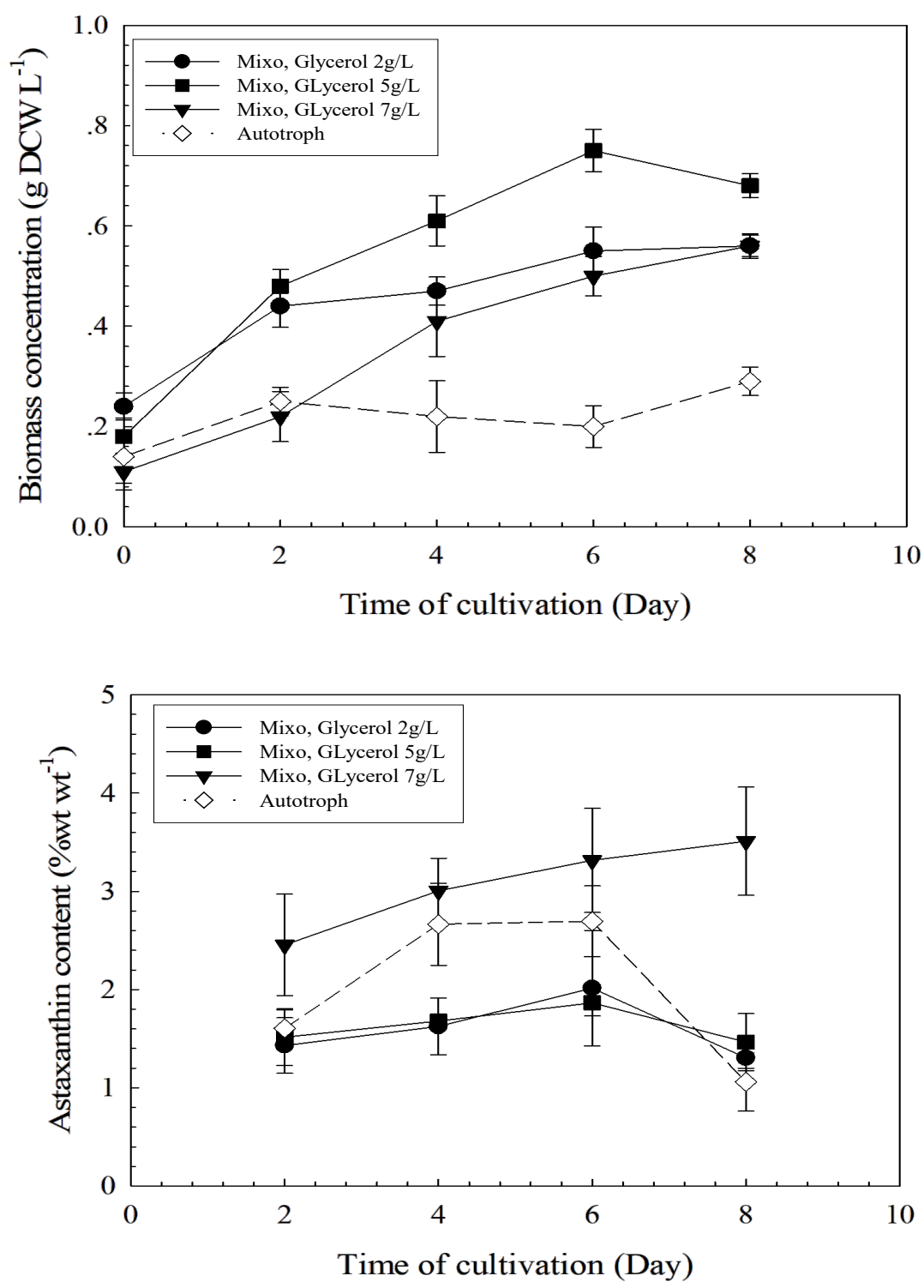

Figure 5 Time profiles of red-stage biomass concentration (A) and astaxanthin content (B) of H. pluvialis cultures, whose green vegetative cells were grown mixotrophically, under refined crude glycerol concentrations of 2, 5 and $7 \mathrm{~g} \mathrm{~L}^{-1}$, and photoautotrophically. 

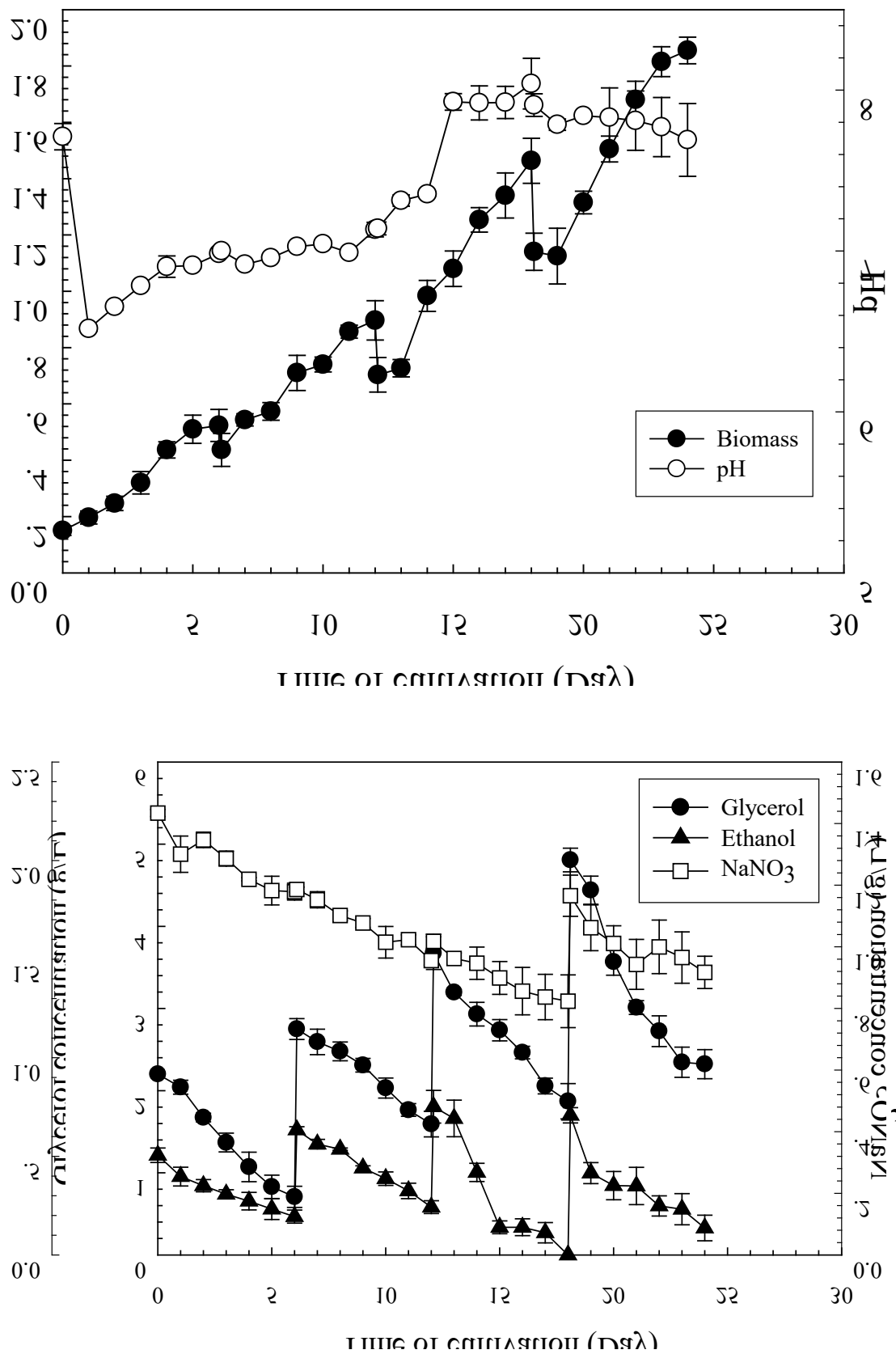

Figure 6 Time profiles of biomass concentration and medium $\mathrm{pH}(\mathbf{A})$ and substrate concentration $(\mathbf{B})$ of H. pluvialis culture, grown mixotrophically using repeated fed-batch operation with the supplementation of 2 g refined crude glycerol $\mathrm{L}^{-1}$ every 6 days. 


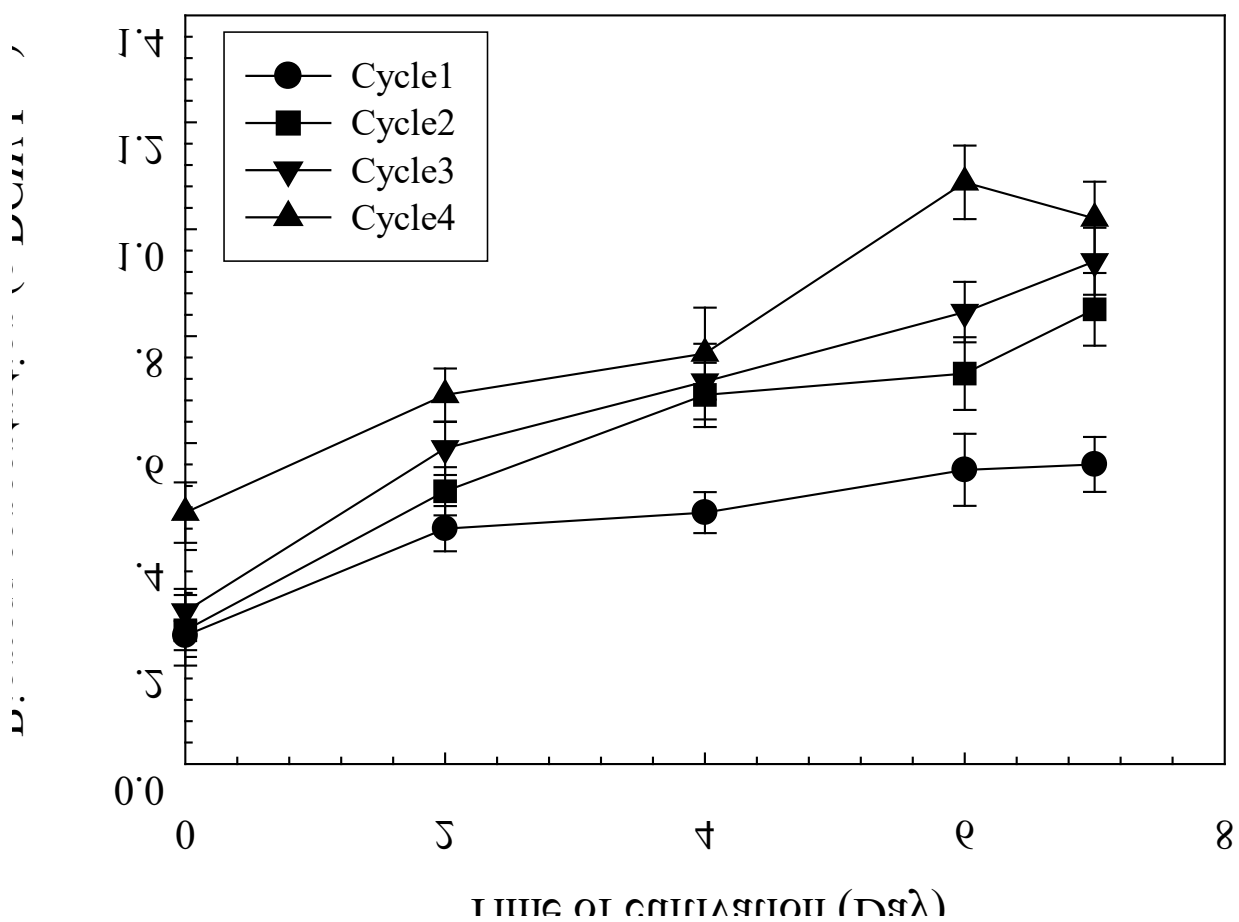

Figure 7 Time profile of red-stage biomass concentration of $H$. pluvialis cultures, whose green vegetative cells were taken from each cycle of mixotrophic and repeated fed-batch cultivation.

Table 3 Performance of mixotrophic repeated fed-batch cultivation in biomass and ATX productions of H. pluvialis.

\begin{tabular}{|c|c|c|c|c|}
\hline \multirow{2}{*}{ Parameters } & \multicolumn{4}{|c|}{ Cycle } \\
\hline & 1 & 2 & 3 & 4 \\
\hline \multicolumn{5}{|c|}{ Green-stage } \\
\hline $\mathrm{X}_{\mathrm{m}}\left(\mathrm{g} \mathrm{DCW} \mathrm{L}^{-1}\right)$ & $0.52 \pm 0.06$ & $0.90 \pm 0.06$ & $1.46 \pm 0.08$ & $1.85 \pm 0.06$ \\
\hline$\Delta \mathrm{X}\left(\mathrm{g} \mathrm{DCW} \mathrm{L}^{-1}\right)$ & $0.37 \pm 0.06$ & $0.46 \pm 0.06$ & $0.76 \pm 0.08$ & $0.71 \pm 0.06$ \\
\hline$\mu\left(\right.$ day $\left.^{-1}\right)$ & $0.22 \pm 0.01$ & $0.12 \pm 0.01$ & $0.13 \pm 0.00$ & $0.12 \pm 0.01$ \\
\hline$\% \mathrm{NaNO}_{3}$ consumption & $17.8 \pm 1.2$ & $19.4 \pm 0.2$ & $19.1 \pm 6.7$ & $21.3 \pm 5.9$ \\
\hline$\%$ Glycerol consumption & $67.8 \pm 5.1$ & $42.1 \pm 1.9$ & $49.1 \pm 2.0$ & $51.7 \pm 13.2$ \\
\hline$\%$ Ethanol consumption & $61.6 \pm 2.5$ & $61.7 \pm 4.4$ & $100.0 \pm 0.0$ & $80.7 \pm 29.9$ \\
\hline \multicolumn{5}{|c|}{ Red-stage } \\
\hline $\mathrm{X}_{\mathrm{m}}\left(\mathrm{g} \mathrm{DCW} \mathrm{L}^{-1}\right)$ & $0.55 \pm 0.06$ & $0.73 \pm 0.07$ & $0.85 \pm 0.06$ & $1.09 \pm 0.07$ \\
\hline$\Delta \mathrm{X}\left(\mathrm{g} \mathrm{DCW} \mathrm{L}^{-1}\right)$ & $0.31 \pm 0.03$ & $0.48 \pm 0.01$ & $0.56 \pm 0.02$ & $0.62 \pm 0.00$ \\
\hline ATX content $(\% \mathrm{wt})$ & $2.0 \pm 0.6$ & $2.5 \pm 0.3$ & $3.0 \pm 0.1$ & $2.3 \pm 0.2$ \\
\hline
\end{tabular}

Data shown are the mean $\pm \mathrm{SD}$ of 3 independent measurements. 
From Figure 6A, the growth profile of the H. pluvialis culture cultivated under $2 \mathrm{~g}$ glycerol L ${ }^{-1}$ and repeated fed-batch operation appeared to be in an exponential phase throughout the entire experiment operation. During cycle 1, the microalgal biomass concentration increased from its initial value of $0.15 \pm$ $0.02 \mathrm{~g} \mathrm{DCW} \mathrm{L}^{-1}$ to reach $0.52 \pm 0.06 \mathrm{~g} \mathrm{DCW} \mathrm{L}^{-1}$ on day 6 , expressing $\mu$ of $0.22 \pm 0.01$ day $^{-1}$ (Table 3) .This observation is highly consistent with that of our batch cultivation under the same initial refined crude glycerol concentration (Table 2). After every replacement of liquid culture with feeding medium, the biomass concentration immediately dropped, due to dilution, before rising again and reaching peaks of $0.90 \pm 0.06,1.46 \pm 0.08$ and $1.86 \pm 0.06 \mathrm{~g} \mathrm{DCW} \mathrm{L}^{-1}$ at the end of cycles 2,3 and 4 , respectively. The value of $\mu$ during cycles 2, 3 and 4 were similar, but around 1.8-fold lower than that of cycle 1 (Table 3). This reduction could be caused by poorer light penetration, generally observed under denser culture $[76,88,89]$. In comparison with our batch cultivations, the repeated fed-batch operation was demonstrated to be a superior means of extending the microalgal exponential growth from day 5 , previously observed in batch operation, to day 24, and significantly enhancing biomass production by around 2.6-fold. This achievement was similar to previous reports $[36,72,73]$ by, and mainly due, to the ability of fed-batch operation to replenish critical media components, and thereby support further cell growth and delay the onset of cell death. With respect to medium $\mathrm{pH}$ (Figure 6A), its profile was similar to that in the batch culture. Specifically, after a $\mathrm{pH}$ drop from its initial value of $7.71 \pm 0.08$ to $6.52 \pm 0.02$ on day 1 , it then increased to a steady value in the range $7.69 \pm 0.23-8.04 \pm 0.16$ on day 15 and remained steady at this level. Regarding substrate profiles (Figure 6B), an almost complete use of ethanol at the end of each cycle was clearly seen, while an excess amount of both glycerol and nitrate lasted throughout the experimental duration. Numerically, from Table 3, total ethanol consumption by $H$. pluvialis of cycles 1 and 2 were similar to that observed from batch cultivation (Table 2) ( 61\%), before reaching 100 and $80.7 \%$ in cycles 3 and 4, respectively. On the other hand, glycerol consumption was found to reach its highest value of $67.8 \%$ in cycle 1 , before falling down to $42.1 \%$ (cycle 2 ) and then climbing up to 49.1 and $51.7 \%$ for the last 2 cycles. For nitrate consumption, the value remained relatively constant in the range $17.8-21.3 \%$ throughout the 4 cycles. Furthermore, this study also investigated the effect of fedbatch growth cycle upon ATX production of H. pluvialis (Figure 7 and Table 3). The highest increment in biomass concentration $\left(0.62 \mathrm{~g} \mathrm{DCW} \mathrm{L}^{-1}\right)$ was observed from cycle 4 vegetative cells. With increasing cycle number, the biomass increment increased. On the other hand, the highest ATX accumulation $(3.0 \pm$ $0.1 \% \mathrm{wt}$ ) was found in microalgal culture from cycle 3 . These variations may have resulted from different life phases of green vegetative cells from each fed-batch cycle that were used for the subsequent ATX induction [90]. Furthermore, other promising cultivation methods could be attempted in order to optimally promote biomass and ATX production during red-stage of $H$. pluvialis, including 3-stage production process [73], stepwise illumination [35], optimized media [84], optimal growth phase for inductive stage [90] or the use of mutant strain [91].

\section{Conclusions}

In this study, although the direct utilization of crude glycerol as a carbon source resulted in $H$. pluvialis growth cessation, its refined state proved to practically facilitate the mixotrophic batch cultivation of the microalga, which resulted in 1.2 - 1.4-fold higher biomass production than that of photoautotrophic conditions. The highest increment in green-stage biomass production, as well as redstage ATX accumulation, were observed from cultures supplemented with $7 \mathrm{~g}$ refined crude glycerol $\mathrm{L}^{-1}$. When the operation was changed from batch to repeated fed-batch, an enhancement in green-stage biomass production (2.6-fold) was observed, thereby proving the latter to be a superior method for microalgal cultivation. Although further studies have to be carried out in order to optimize the production process, our results could be used as the preliminary for future investigations or process scale-up. 


\section{Acknowledgements}

This work was supported by the Higher Education Research Promotion and National Research University Project of Thailand, Office of the Higher Education Commission [grant number 2559A30562007, 2016].

\section{References}

[1] N Shimidzu, M Goto and W Miki, Carotenoids as singlet oxygen quenchers in marine organisms. Fish Sci. 1996; 62, 134-7.

[2] Y Naguib. Antioxidant activities of astaxanthin and related carotenoids. J. Agric. Food Chem. 2000; 48, 1150-4.

[3] T Tanaka, Y Morishita, M Suzui, T Kojima, A Okumura and H Mori. Chemoprevention of mouse urinary bladder carcinogenesis by the naturally occurring carotenoid astaxanthin. Carcinogenesis 1994; 15, 15-9.

[4] R Nakao, OL Nelson, JS Park, BD Mathison, PA Thompson and BP Chew. Effect of dietary astaxanthin at different stages of mammary tumor initiation in BALB/c mice. Anticancer Res. 2010; 30, 2171-5.

[5] CM Partland. Effects of astaxanthin on the proliferation and migration of breast cancer cells in vitro. Antioxidants 2018; 7, 135.

[6] H Jyonouchi, L Zhang, M Gross and $\mathrm{Y}$ Tomita. Immunomodulating actions of carotenoids: Enhancement of in vivo and in vitro antibody production to T-dependent antigens. Nutr. Cancer. 1994; 21, 47-58.

[7] JS Park, JH Chyun, YK Kim, LL Line and BP Chew. Astaxanthin decreased oxidative stress and inflammation and enhanced immune response in humans. Nutr. Metab. 2010; 7, 18.

[8] N Ito, S Seki and F Ueda. The protective role of astaxanthin for UV-induced skin deterioration in healthy people: A randomized, double-blind, placebo-controlled trial. Nutrients 2018; 10,817.

[9] M Kobayashi and T Okada. Protective role of astaxanthin against u.v.-B irradiation in the green alga Haematococcus pluvialis. Biotechnol. Lett. 2000; 22: 177-81.

[10] T Komatsu, S Sasaki, Y Manabe, T Hirata and T Sugawara. Preventive effect of dietary astaxanthin on UVA-induced skin photoaging in hairless mice. PloS One 2017; 12, e0171178.

[11] M Guerin, ME Huntley and M Olaizola. Haematococcus astaxanthin: Applications for human health and nutrition. Trends Biotechnol. 2003; 21, 210-6.

[12] S Davinelli, M Nielsen and G Scapagnini. Astaxanthin in skin health, repair, and disease: A comprehensive review. Nutrients 2018; 10, 522.

[13] R Fassett and J Coombes. Astaxanthin: A potential therapeutic agent in cardiovascular disease. Mar. Drugs 2011; 9, 447-65.

[14] RT Lorenz and GR Cysewski. Commercial potential for Haematococcus microalgae as a natural source of astaxanthin. Trends Biotechnol. 2000; 18, 160-7.

[15] I Higuera-Ciapara, L Félix-Valenzuela and FM Goycoolea. Astaxanthin: A review of its chemistry and applications. Crit. Rev. Food Sci. Nutr. 2006; 46, 185-96.

[16] Astaxanthin Market Analysis By Source (Natural [Yeast, Krill/Shrimp, Microalgae] and Synthetic). By Product (Dried Biomass/Powder, Oil, Soft gels, Liquid), By Application, and Segment Forecasts, 2018 - 2025. Grand View Research, 2017, p. 96.

[17] M Koller, A Muhr and G Braunegg. Microalgae as versatile cellular factories for valued products. Algal Res. A 2014; 6, 52-63.

[18] J Li, D Zhu, J Niu, S Shen and G Wang. An economic assessment of astaxanthin production by large scale cultivation of Haematococcus pluvialis. Biotechnol. Adv. 2011; 29, 568-74.

[19] B Capelli, D Bagchi and G Cysewski. Synthetic astaxanthin is significantly inferior to algal-based astaxanthin as an antioxidant and may not be suitable as a human nutraceutical supplement. Nutrafoods 2013; 12, 145-52. 
[20] M Wayama, S Ota, H Matsuura, N Nango, A Hirata and S Kawanoet. Three-dimensional ultrastructural study of oil and astaxanthin accumulation during encystment in the green alga Haematococcus pluvialis. PloS One 2013; 8, e53618.

[21] U Tripathi, R Sarada and R Gokare. Production of astaxanthin in Haematococcus pluvialis cultured in various media. Bioresour. Technol. 1999; 68, 197-9.

[22] M Olaizola and M Huntley. Recent advances in commercial production of astaxanthin from microalgae. Vol. 9. Science Publishers, New Hampshire, 2003, p. 143-64.

[23] M Wan, D Hou, Y Li, J Fan, J Huang, S Liang, W Wang, R Pan, J Wang and S Li. The effective photoinduction of Haematococcus pluvialis for accumulating astaxanthin with attached cultivation. Bioresour. Technol. 2014; 163, 26-32.

[24] SK Saha, E McHugh, J Hayes, S Moane, D Walsh and P Murray. Effect of various stress-regulatory factors on biomass and lipid production in microalga Haematococcus pluvialis. Bioresour. Technol. 2013; 128, 118-24.

[25] J Park, SP Choi, ME Hong and SJ Sim. Enhanced astaxanthin production from microalga, Haematococcus pluvialis by two-stage perfusion culture with stepwise light irradiation. Bioprocess Biosyst. Eng. 2014; 37, 2039-47.

[26] B Wang, A Zarka, A Trebst and S Boussiba. Astaxanthin accumulation in Haematococcus pluvialis (Chlorophyceae) as an active photoprotective process under high Irradiance. J. Psychol. 2003; 39, 1116-24.

[27] M Kobayashi, Y Kurimura and Y Tsuji. Light-independent, astaxanthin production by the green microalga Haematococcus pluvialis under salt stress. Biotechnol. Lett. 1997; 19, 507-9.

[28] N Hata, JC Ogbonna, Y Hasegawa, H Taroda and H Tanaka. Production of astaxanthin by Haematococcus pluvialis in a sequential heterotrophic-photoautotrophic culture. J. Appl. Phycol. 2001; 13, 395-402.

[29] SW Hwang, HI Choi and SJ Sim. Acidic cultivation of Haematococcus pluvialis for improved astaxanthin production in the presence of a lethal fungus. Bioresour. Technol. 2019; 278, 138-44.

[30] A Tjahjono, Y Hayama, T Kakizono, Y Terada, N Nishio and S Nagai. Hyper-accumulation of astaxanthin in a green alga Haematococcus pluvialis at elevated temperatures. Biotechnol. Lett. 1994; 16, 133-8.

[31] M Wan, J Zhang, D Hou, J Fan, Y Li, J Huang and J Wang, The effect of temperature on cell growth and astaxanthin accumulation of Haematococcus pluvialis during a light-dark cyclic cultivation. Bioresour. Technol. 2014; 167, 276-83.

[32] M Zhekisheva, S Boussiba, IK Goldberg, A Zarka and Z Cohen. Accumulation of oleic acid in Haematococcus pluvialis (Chlorophyceae) under nitrogen starvation or high light is correlated with that of astaxanthin esters. J. Phycol. 2002; 38, 325-31.

[33] S Boussiba, W Bing, JP Yuan, A Zarka and F Chen. Changes in pigments profile in the green alga Haeamtococcus pluvialis exposed to environmental stresses. Biotechnol. Lett. 1999; 21, 601-4.

[34] A Lababpour, K Hada, K Shimahara, T Katsuda and S Katoh. Effects of nutrient supply methods and illumination with blue light emitting diodes (LEDs) on astaxanthin production by Haematococcus pluvialis. J. Biosci. Bioeng. 2004. 98, 452-6.

[35] CD Kang, SJ Han, SP Choi and SJ Sim. Fed-batch culture of astaxanthin-rich Haematococcus pluvialis by exponential nutrient feeding and stepwise light supplementation. Bioprocess Biosyst. Eng. 2009; 33,133.

[36] A Lababpour, K Shimahara, K Hada, Y Kyoui, T Katsuda, and S Katoh. Fed-batch culture under illumination with blue light emitting diodes (LEDs) for astaxanthin production by Haematococcus pluvialis. J. Biosci. Bioeng. 2005; 100, 339-42.

[37] R Ranjbar, R Inoue, H Shiraishi, T Katsuda and S Katoh. High efficiency production of astaxanthin by autotrophic cultivation of Haematococcus pluvialis in a bubble column photobioreactor. Biochem. Eng. J. 2008; 39, 575-80.

[38] M Kobayashi, T Kakizono, K Yamaguchi, N Nishio and S Nagai. Growth and astaxanthin formation of Haematococcus pluvialis in heterotrophic and mixotrophic conditions. J. Ferment Bioeng. 1992; 74, 17-20. 
[39] M Olaizola. Commercial production of astaxanthin from Haematococcus pluvialis using 25,000liter outdoor photobioreactors. J. Appl. Phycol. 2000; 12, 499-506.

[40] BY Zhang, YH Geng, ZK Li, HJ Hu and YG Li. Production of astaxanthin from Haematococcus in open pond by two-stage growth one-step process. Aquaculture 2009; 295, 275-81.

[41] JJ Yoo, SP Choi, BW Kim and SJ Sim. Optimal design of scalable photo-bioreactor for phototropic culturing of Haematococcus pluvialis. Bioprocess Biosyst. Eng. 2012; 35, 309-15.

[42] J Fábregas, A Otero, A Maseda and A Domínguez. Two-stage cultures for the production of Astaxanthin from Haematococcus pluvialis. J. Biotechnol. 2001; 89, 65-71.

[43] G Cysewski and R Lorenz. Industrial production of microalgal cell-mass and secondary productsspecies of high potential: Haematococcus. John Wiley \& Sons, 2007, p. 281-8.

[44] PP López, SG Garcia, C Jeffryes, SN Agathos, E Mchugh, DJ Walsh, PM Murray, S Moane, G Feijoo and MT Moreira. Life cycle assessment of the production of the red antioxidant carotenoid astaxanthin by microalgae: From lab to pilot scale. J. Clean. Prod. 2014; 64, 332-44.

[45] MMR Shah, Y Liang, JJ Cheng and M Daroch. Astaxanthin-producing green microalga Haematococcus pluvialis: From single cell to high value commercial products. Front. Plant Sci. 2016; 7, 531.

[46] G Panis and JR Carreón. Commercial astaxanthin production derived by green alga Haematococcus pluvialis: A microalgae process model and a techno-economic assessment all through production line. Algal Res. 2016; 18, 175-90.

[47] F Yang, M Hanna and R Sun. Value-added uses for crude glycerol: A byproduct of biodiesel production. Biotechnol. Biofuels 2012; 5, 13.

[48] S Chozhavendhan, RP Kumar, S Elavazhagan, B Barathiraja, M Jayakumar and SJ Varjani. Utilization of crude glycerol from biodiesel industry for the production of value-added bioproducts. In: R Singhania, R Agarwal, R Kumar, R Sukumaran (Eds.). Waste to wealth. Springer, Singapore, 2018, p. 65-82.

[49] LR Kumar, SK Yellapu, RD Tyagi and X Zhang. A review on variation in crude glycerol composition, bio-valorization of crude and purified glycerol as carbon source for lipid production. Bioresour. Technol. 2019; 293, 122155.

[50] SS Win and TA Trabold. Sustainable waste-to-energy technologies: Transesterification. In: TA Trabold and CW Babbitt (Eds.). Sustainable food waste-to-energy systems. $1^{\text {st }}$ ed. Academic Press, United States, 2018, p. 89-109.

[51] JCJ Bart, N Palmeri and S Cavallaro. Valorisation of the glycerol by-product from biodiesel production. In: JCJ Bart, N Palmeri and S Cavallaro (Eds.). Biodiesel science and technology. $1^{\text {st }}$ ed. Woodhead Publishing, UK, 2010, p. 571-624.

[52] M Pagliaro and M Rossi. The Future of Glycerol. Royal Society of Chemistry, 2008.

[53] M Anitha, SK Kamarudin and NT Kofli. The potential of glycerol as a value-added commodity. Chem. Eng. J. 2016; 295, 119-30.

[54] M McCoy. An unlikely impact. Chem. Eng. News. 2005; 83, 19-20.

[55] VK Garlapati, U Shankar and A Budhiraja. Bioconversion technologies of crude glycerol to value added industrial products. Biotechnol. Rep. 2016; 9, 9-14.

[56] G Bagnato, A Iulianelli, A Sanna and A Basile. Membranes glycerol production and transformation: A critical review with particular emphasis on glycerol reforming reaction for producing hydrogen in conventional and membrane reactors. Membranes 2017; 7, 7.

[57] H Choi and SW Yu. Influence of crude glycerol on the biomass and lipid content of microalgae. Biotechnol. Biotechnol. Equip. 2015; 29, 506-13.

[58] R Katiyar, BR Gurjar, RK Bharti, A Kumar, S Biswas and V Pruthi. Heterotrophic cultivation of microalgae in photobioreactor using low cost crude glycerol for enhanced biodiesel production. Renew. Energ. 2017; 113, 1359-65.

[59] YH Chen and TH Walker. Biomass and lipid production of heterotrophic microalgae Chlorella protothecoides by using biodiesel-derived crude glycerol. Biotechnol. Lett. 2011; 33, 1973. 
[60] M Bindea, B Rusu, A Rusu, M Trif, LF Leopold, F Dulf and DC Vodnar. Valorification of crude glycerol for pure fractions of docosahexaenoic acid and $\beta$-carotene production by using Schizochytrium limacinum and Blakeslea trispora. Microb. Cell Fact. 2018; 17, 97.

[61] WB Kong, H Yang, YT Cao, H Song, SF Hua and CG Xia. Effect of glycerol and glucose on the enhancement of biomass, Lipid and soluble carbohydrate production by Chlorella vulgaris in mixotrophic culture. Food Technol. Biotechnol. 2013, 51, 62-9.

[62] T Göksan, İ Ak and S Gokpinar. An alternative approach to the traditional mixotrophic cultures of Haematococcus pluvialis flotow (Chlorophyceae). J. Microbiol. Biotechnol. 2010; 20, 1276-82.

[63] M Orosa, D Franqueira, A Cid and J Abalde. Analysis and enhancement of astaxanthin accumulation in Haematococcus pluvialis. Bioresour. Technol. 2005; 96, 373-8.

[64] S Oncel, D Franqueira, A Cid and J Abalde. Comparison of different cultivation modes and light intensities using mono-cultures and co-cultures of Haematococcus pluvialis and Chlorella zofingiensis. J. Chem. Technol. Biotechnol. 2011; 86, 414-20.

[65] M Kobayashi, T Kakizono and S Nagai. Astaxanthin production by a green alga Haematococcus pluvialis accompanied with morphological changes in acetate media. J. Ferment. Bioeng. 1991; 71, 335-9.

[66] M Zych, A Stolarczyk, K Maca, A Banaś, KT Pabis, A Kapuścik, S Klasik and J Burczyk. Assimilation of various organic carbon sources by Haematococcus strains. Acta Agron. Hung. 2009; 57, 231-7.

[67] C Hagen, K Grünewald, M Xyländer and E Rothe. Effect of cultivation parameters on growth and pigment biosynthesis in flagellated cells of Haematococcus pluvialis. J. Appl. Phycol. 2001; 13, 79-87.

[68] V Andruleviciute, V Makareviciene, V Skorupskaite and M Gumbyte. Biomass and oil content of Chlorella sp., Haematococcus sp., Nannochloris sp. and Scenedesmus sp. under mixotrophic growth conditions in the presence of technical glycerol. J. Appl. Phycol. 2014; 26, 83-90.

[69] M Orosa, D Franqueira, A Cid and J Abalde. Carotenoid accumulation in Haematococcus pluvialis in mixotrophic growth. Biotechnol. Lett. 2001; 23, 373-8.

[70] M Kobayashi, T Kakizono and S Nagai. Enhanced carotenoid biosynthesis by oxidative stress in acetate-induced cyst cells of a green unicellular alga Haematococcus pluvialis. Appl. Environ. Microbiol. 1993; 59, 867-73.

[71] M Azizi, MA Hejazi and M Hashemi. Supplementation with polyalcohols and sequential mixotrophy dilution photoinduction strategy boost the accumulation of astaxanthin by Haematococcus pluvialis. Aquaculture. 2019; 511, 734225.

[72] XW Zhang, XD Gong and F Chen. Kinetic models for astaxanthin production by high cell density mixotrophic culture of the microalga Haematococcus pluvialis. J. Ind. Microbiol. Biotechnol. 1999; 23, 691-6.

[73] H Sun, B Guan, Q Kong, Z Geng and N Wang. Repeated cultivation: Non-cell disruption extraction of astaxanthin for Haematococcus pluvialis. Sci. Rep. 2016; 6, 20578.

[74] R Manosak, S Limpattayanate and M Hunsom. Sequential-refining of crude glycerol derived from waste used-oil methyl ester plant via a combined process of chemical and adsorption. Fuel Process. Technol. 2011; 92, 92-9.

[75] S Boussiba and A Vonshak. Astaxanthin Accumulation in the Green Alga Haematococcus pluvialis ${ }^{1}$. Plant Cell Physiol. 1991; 32, 1077-82.

[76] J Wang, D Han, MR Sommerfeld, C Lu and Q Hu. Effect of initial biomass density on growth and astaxanthin production of Haematococcus pluvialis in an outdoor photobioreactor. J. Appl. Phycol. 2013; 25, 253-60.

[77] R Sarada, R Vidhyavathi, D Usha and GA Ravishankar. An efficient method for extraction of astaxanthin from green alga Haematococcus pluvialis. J. Agric. Food Chem. 2006; 54, 7585-8.

[78] HJ Choi and SW Yu. Influence of crude glycerol on the biomass and lipid content of microalgae. Biotechnol. Biotechnol. Equip. 2015; 29, 506-13.

[79] S Ethier, K Woisard, D Vaughan and Z Wen. Continuous culture of the microalgae Schizochytrium limacinum on biodiesel-derived crude glycerol for producing docosahexaenoic acid. Bioresour. Technol. 2011; 102, 88-93. 
[80] D Samul, K Leja and W Grajek. Impurities of crude glycerol and their effect on metabolite production. Ann. Microbiol. 2014; 64, 891-8.

[81] J Wang, E Atolia, B Hua, Y Savir, RE Chong and M Springer. Natural variation in preparation for nutrient depletion reveals a cost-benefit tradeoff. PloS Biol. 2015; 13, e1002041.

[82] D Chu. and D Barnes. The lag-phase during diauxic growth is a trade-off between fast adaptation and high growth rate. Sci. Rep. 2016; 6, 25191.

[83] C Zhang, L Zhang and J Liu. Exogenous sodium acetate enhances astaxanthin accumulation and photoprotection in Haematococcus pluvialis at the non-motile stage. J. Appl. Phycol. 2019; 31, 10018.

[84] CD Kang, JS Lee, TH Park and SJ Sim. Comparison of heterotrophic and photoautotrophic induction on astaxanthin production by Haematococcus pluvialis. Appl. Microbiol. Biotechnol. 2005; 68, 23741

[85] T Xi, DG Kim, SW Roh, JS Choi and YE Choi. Enhancement of astaxanthin production using Haematococcus pluvialis with novel LED wavelength shift strategy. Appl. Microbiol. Biotechnol. 2016; 100, 6231-8.

[86] AE Solovchenk., Recent breakthroughs in the biology of astaxanthin accumulation by microalgal cell. Photosynth. Res. 2015; 125, 437-49.

[87] Z Wen, Z Liu, Y Hou, C Liu, F Gao, Y Zheng and F Chen. Ethanol induced astaxanthin accumulation and transcriptional expression of carotenogenic genes in Haematococcus pluvialis. Enzyme Microb. Technol. 2015; 78, 10-7.

[88] D Zhang, P Dechatiwongse, EA del Rio-Chanona, GC Maitland, K Hellgardt and VS Vassiliadis. Dynamic modelling of high biomass density cultivation and biohydrogen production in different scales of flat plate photobioreactors. Biotechnol. Bioeng. 2015; 112, 2429-38.

[89] D Zhang, P Dechatiwongse, EA del Rio-Chanona, GC Maitland, K Hellgardt and VS Vassiliadis. Modelling of light and temperature influences on cyanobacterial growth and biohydrogen production. Algal Res. 2015; 9, 263-74.

[90] YE Choi, YS Yun, JM Park and JW Yang. Determination of the time transferring cells for astaxanthin production considering two-stage process of Haematococcus pluvialis cultivation. Bioresour. Technol. 2011; 102, 11249-53.

[91] ME Hong, SP Choi, YI Park, YK Kim, WS Chang, BW Kim and SJ Sim. Astaxanthin production by a highly photosensitive Haematococcus mutant. Process Biochem. 2012; 47, 1972-9. 